\title{
Medical conditions and medication use in adults with Down syndrome: A descriptive analysis
}

\author{
Gerard Kerins ${ }^{1,2}$, Kimberly Petrovic ${ }^{1,3}$, Mary Beth Bruder' and Cynthia Gruman ${ }^{4}$
}

Background: We examined the presence of medical conditions and medication use within a sample of adults with Down syndrome. Methods: Retrospective chart review using a sample of 141 adults with Down syndrome and age range of 30 to 65 years. Results: We identify 23 categories of commonly occurring medical conditions and 24 categories of medications used by adults with Down syndrome. Conclusion: Approximately $75 \%$ of older adults with Down syndrome in our sample experience memory loss and dementia. Hypothyroidism, seizures, and skin problems also occur commonly. The prevalence of cancer (i.e., solid tumours) and hypertension is extremely low. Older adults with Down syndrome use anticonvulsant more often than younger adults with Down syndrome. The use of multivitamins and medications such as pain relievers, prophylactic antibiotics, and topical ointments is common.

(1) University of Connecticut Health Center, A.J. Pappanikou Center for Developmental Disabilities, Farmington CT, USA

(2) Hospital of Saint Raphael, New Haven CT, USA

(3) University of Connecticut, School of Nursing, Storrs CT, USA

(4) University of Connecticut Health Center, Center on Aging, Farmington CT, USA

Correspondence to Kimberly Petrovic $\cdot$ e-mail: kimberly.petrovic@uconn.edu

doi:10.3104/reports.2009

() 2007 The Authors. Journal Compilation @ 2008 Down

Syndrome Education International.
Adults with intellectual disabilities such as Down syndrome are experiencing simultaneous increases in life expectancy and the prevalence of certain medical condition $s^{[1]}$, including hypothyroidism ${ }^{[2]}$ and sleep apnoea ${ }^{[3]}$. Premature aging is not uncommon for older adults with Down syndrome $\mathrm{e}^{[4]}$. Dementia is common among adults with Down syndrome $e^{[5]}$, as dementia and neuropsychological decline may accompany increased life expectancy for adults with Down syndrome. Prevalence data available with regard to dementia in adults with Down syndrome suggest that dementia occurs in $36-66 \%$ of adults between the ages of 50 to 59 years old and 9 $11 \%$ of adults between the ages of 40 to 49 years old $^{[6,7,8]}$. Additionally, increases in the use of certain medications are believed to occur among adults with Down syndrome when compared to adults without Down syndrome. Yet, little information has been published with regard to the types of medications and their indications for use.

Numerous medical conditions are believed to be associated with the aging of adults with Down syndrome. Generalised lens opacities such as total cataract ${ }^{[9]}$ may result from increased amounts of free radical reactions in adults with
Down syndrome ${ }^{[10]}$. Hypercholesterolaemia is not uncommon in older adults with Down syndrome $^{[11]}$ and may be associated with family history ${ }^{[12,13]}$. Hypothyroidism may develop secondary to autoimmune thyroiditis ${ }^{[2]}$. Obstructive sleep apnoea may result from physiological and anatomical abnormalities in adults with Down syndrome ${ }^{[3]}$. Adults with Down syndrome experience limitations in cardio-respiratory capacity $^{\left[{ }^{[14]} \text {, osteoporosis }\right.}{ }^{[15]}$, and late-onset seizures ${ }^{[16]}$. In fact, late-onset seizures are thought to occur in 75 - $85 \%$ of adults with Down syndrome who are affected by Alzheimer's disease after the age of $50^{[16]}$.

Adults with Down syndrome are susceptible to the presence of neuropsychological conditions such as anxiety, depression, and dementia. Due to the progressive, age-dependent nature of Alzheimer's disease pathology involving the deposition of diffuse amyloid plaques that accumulate in the brains of adults with Down syndrome who are older than the age of $30^{[5,17]}$, adults with Down syndrome are at heightened risk for developing Alzheimer's disease ${ }^{[5,18]}$. Research shows that the onset of Alzheimer's disease takes place at approximately 40 years of age ${ }^{[19]}$, an age which is considered 'young' by many standards 
in our society. Dementia is difficult to diagnose in adults with Down syndrome who concurrently experience co-morbid states ${ }^{[20]}$. Further, the rates of co-morbidities are high for adults with Alzheimer's disease ${ }^{[21]}$, thus creating a cycle in which dementia is not readily assessed and the prevention of neuropsychological decline takes on greater complexity.

\section{Purpose of this paper}

Based on this information, the need exists for additional research that examines the aging process of adults with Down syndrome. This includes an examination of the presence of medical conditions affecting adults with Down syndrome as well as the medications prescribed for the treatment of these conditions. The purpose of this paper is to investigate the following two questions:

1. What are the medical conditions that commonly characterise adults with Down syndrome?

2. What types of medications are commonly used by adults with Down syndrome?

\section{Methods}

A retrospective chart review was conducted of 187 adults ages 30 - 65 and with documented intellectual disability. All adults were established patients at a major teaching hospital in a metropolitan area of the United States in which faculty (e.g., certain geriatricians) possessed extensive experience in working with adults with intellectual disabilities. As a result, adults with intellectual disabilities were referred to the hospital from throughout the entire statewide region for geriatric evaluations that included specialised Down Syndrome Mental Status testing and physical assessments.

Approval from the Institutional Review Board of the University School of Medicine was obtained prior to carrying out this study. Data obtained from the chart review were entered into a software spreadsheet created using the Statistical Package for the Social Sciences (SPSS). Identifiers were removed for each chart. No medical record numbers or names of individuals were obtained or entered into the SPSS database.

Within the sample of 187 adults with intellectual disabilities, a total of 141 had a diagnosis of Down syndrome. Based upon the literature, categories of age, physical health conditions, neuropsychological conditions, medications, and demographic variables were created. Categories of age include: (1) up to 39 years old, (2) 40-49 years old, (3) 50-59 years old, (4) and 60 years old or older.

A total of 23 categories of medical conditions

\begin{tabular}{|c|c|c|c|c|c|c|}
\hline & \multicolumn{2}{|c|}{ Less than 50 years old } & \multicolumn{2}{|c|}{50 years old or older } & \multicolumn{2}{|c|}{ Total } \\
\hline & number & $\%$ & number & $\%$ & number & $\%$ \\
\hline $\begin{array}{l}\text { Gender of individual: } \\
\text { Male }\end{array}$ & 52 & 67 & 32 & 50 & 84 & 59 \\
\hline $\begin{array}{l}\text { Gender of individual: } \\
\text { Female }\end{array}$ & 25 & 33 & 32 & 50 & 57 & 41 \\
\hline Total & 77 & 100 & 64 & 100 & 141 & 100 \\
\hline
\end{tabular}

\begin{tabular}{|lllllll|}
\hline \multicolumn{4}{c}{ Less than $\mathbf{5 0}$ years old } & $\mathbf{5 0}$ years old or older & \multicolumn{2}{c|}{ Total } \\
\cline { 2 - 6 } & number & $\%$ & number & $\%$ & number & $\%$ \\
\hline
\end{tabular}

\section{Anxiety or depression}

Anxiety:

Depression:

Both:

Neither:

Total:

Other mental illness

Yes:

No:

Total:

Dementia

Yes:

No:

Total:

\section{Arthritis}

Yes:

No:

Total:

Cancer

Yes:

No:

Total:

number $\quad \% \quad$ number $\quad \%$ number

$\%$

Cardiac condition:

Murmur

Yes:

No:

Total:

$\begin{array}{lllll}4 & 5 & 6 & 9 & 10\end{array}$

$17 \quad 22$

$5 \quad 7$

$51 \quad 66$

$77 \quad 100$

50

64

13

0

78
100

25

25
5
101

101
141

\section{Cardiac condition:}

Other

Yes:

No:

Total:

$55 \quad 71$

23

78

30

100

\section{Cataract}

Yes:

No:

Total:

$\begin{array}{rrr}6 & 8 & 12 \\ 71 & 92 & 52 \\ 77 & 100 & 64\end{array}$

52

12

64

81
19

100

107

35

75

25

100

$\begin{array}{llll}12 & 19 & 18 & 13\end{array}$

52

64

13
87

$81 \quad 123$

$100 \quad 141$

87
100

Diagnosed disability of

any type

Yes:

No:

23

1
63

97
100

2
98

3

138

141

2
98

100

Total:

Diverticulosis/

Diverticulitis/

Crohn's disease

Yes:

No:

Total:

10

67

77

13
87

87
100

10

54

16

20

121

141

14

86
100

100

\section{GERD}

(Gastroesophageal

reflux disease)

Yes:

No:

Total:

$\begin{array}{rr}11 & 15 \\ 66 & 85 \\ 77 & 100\end{array}$

9

56

64

5
59
64

Table 2 | Medical condition and age of individual with Down syndrome 


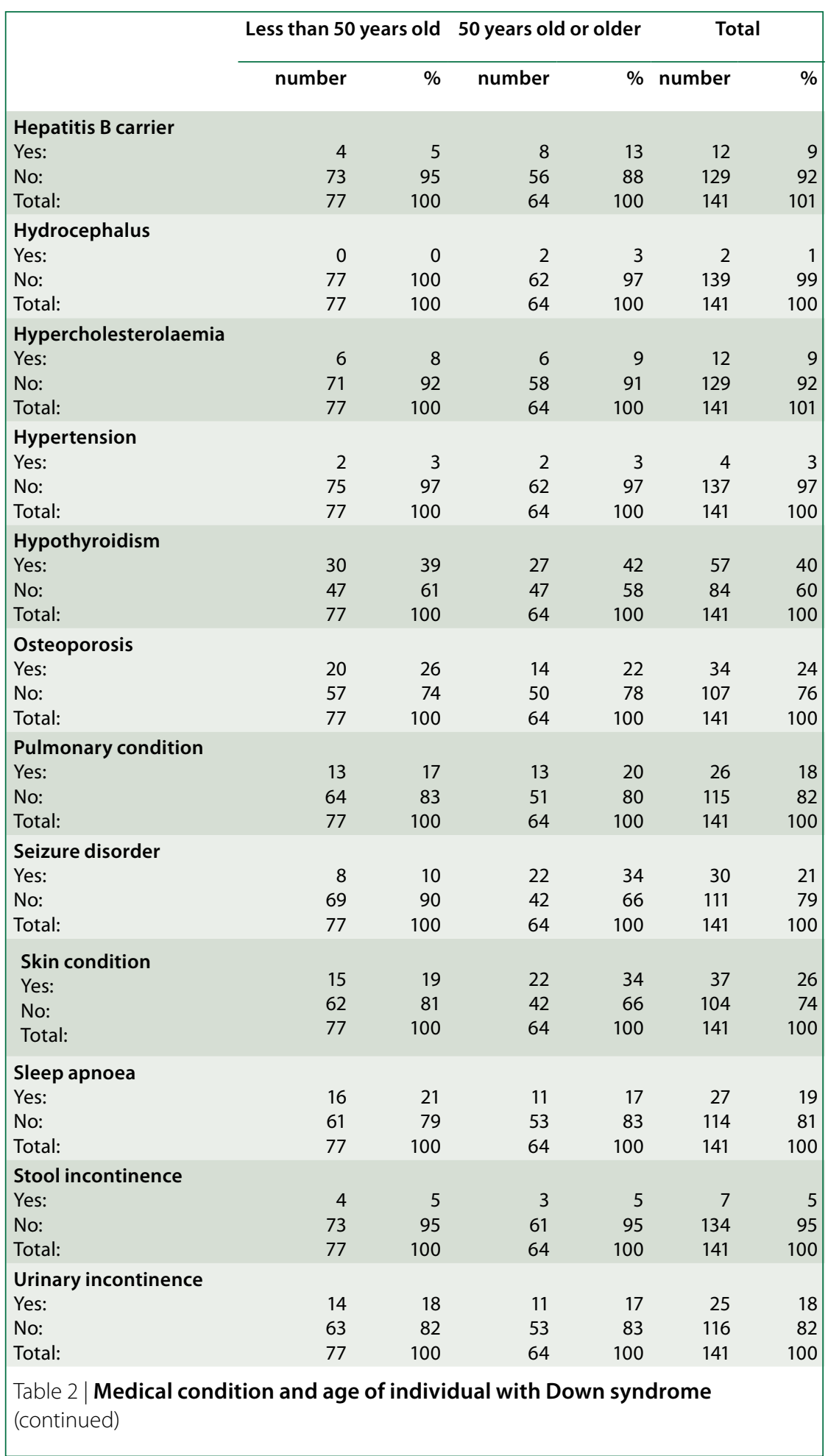

were created. They include: anxiety or depression, other mental illness (e.g., psychosis), dementia, arthritis or other musculoskeletal condition, cancer, cardiac condition (murmur), other cardiac condition (e.g., atrioventricular septal defect/AVSD), cataract, diverticulosis/diverticulitis/Crohn's disease, gastroesophageal reflux disease (GERD), Hepatitis B carrier, hydrocephalus, hypercholesterolemia, hypertension, hypothyroidism, osteoporosis, pulmonary conditions (e.g., asthma, chronic obstructive pulmonary disease, pneumonia), seizure disorder, skin con- ditions, (obstructive) sleep apnoea, stool incontinence and urinary incontinence. Likewise, a separate category entitled "diagnosed disability of any type" (e.g., physical, emotional, learning) was developed, as we believe that this category is distinctive and differs conceptually from medical or neuropsychological conditions.

A total of 24 categories of medications were created and reflect prescribed medications only. The categories of medications are: anti-anxiety medications, anticonvulsants, antidepressants, anti-hypertension medications, antipsychotic medications, antispasmodics, cholesterol-lowering medications, cholinesterase inhibitors, Fosamax - or alendronate sodium, a biphosphonate that inhibits bone resorption without impeding mineralisation ${ }^{[22]}$, GERD-related medications (e.g., proton pump inhibitors), hormones (other than thyroid-related hormones), hypothyroidrelated medications, respiratory medications (e.g., metered dose inhalers/MDIs), Vitamin A, Vitamin B12, Vitamin C, Vitamin E, calcium, folic acid, iron, multivitamin, no vitamin/mineral, other vitamin/mineral, and other medications (e.g., pain relievers, prophylactic antibiotics, and topical ointments).

\section{Results}

We examined the presence of medical diagnoses and medication utilisation as they pertain to adults with Down syndrome. Cross-tabulations were performed for all categories of medical conditions and medications. The number of medications used by adults with Down syndrome ranged from 0 - 16 medications per adult.

The data contain multiple cohorts of adults. However, we specifically focused on: (1) adults with Down syndrome $(\mathrm{n}=141)$, and (2) adults without Down syndrome $(n=45)$. The group of adults with Down syndrome was divided into (1) adults under the age of 50 years, known as 'younger adults' $(\mathrm{n}=77)$ and (2) adults 50 years old or older, known as 'older adults' $(n=64)$. The average age of this sample was approximately 51 years old (50.98 years), with a range of 31-65 years. The sample contained greater numbers of men $(n=84)$ as compared to women $(n=57)$. Fewer men were found in the $50+$ group $(n=32)$ than in the group of adults under the age of 50 (n $=52$ ). More women were found in the 50+ group $(n=32)$ as compared to those under the age of 50 years $(n=25)$. (See TABLE 1.)

TABLE 2 contains information pertaining to medical conditions by age group. Regarding the entire sample of adults with Down syndrome, relatively large percentages of dementia (75.9\%), hypothyroidism $(40.4 \%)$, skin conditions $(26.2 \%)$, seizure disorder (21.3\%), and pulmonary conditions 
(18.4\%) exist. Relatively small percentages of hydrocephalus (1.4\%) and hypertension $(2.8 \%)$ exist for this sample of adults with Down syndrome. In both groups, the presence of certain cardiac conditions (e.g., AVSD) and GERD exist in nearly equal percentages $(18.4 \%$ and $14.2 \%$, respectively). Overall, older adults (50+ years old) with Down syndrome experience more instances of all medical conditions except depression, other mental illnesses, cancer, osteoporosis, sleep apnoea, and stool and urinary incontinence as compared to the younger group of adults ( $>50$ years old) with Down syndrome.

Anxiety is present for $9.4 \%$ of older adults with Down syndrome, and dementia occurs in over $80 \%$ of cases in this group. Adults with Down syndrome who are 50 years old or older are found to have higher percentages of arthritis/ other musculoskeletal conditions (19\%), heart murmurs (16\%), and cataracts (14\%) when compared to the younger group. Greater percentages of older adults with Down syndrome experience diverticulosis or a related condition ( $8 \%$ ), are carriers of the Hepatitis B virus (13\%), and have higher cholesterol levels (9\%) as compared to the younger group of adults with Down syndrome. Over $40 \%$ of older adults with Down syndrome experience hypothyroidism, and nearly 35\% have been diagnosed with seizure disorder or skin conditions.

TABLE 3 represents medication use according to the age of adults with Down syndrome. Comparable percentages of younger adults and older adults with Down syndrome use anti-anxiety medications (16\% vs. $16 \%)$, cholesterol-lowering agents ( $9 \%$ vs. $11 \%)$, hormones other than thyroid-related hormones $(13.0 \%$ vs. $14 \%)$, and calcium (30\% vs. $30 \%)$. Less than $10 \%$ of this sample of adults with Down syndrome uses folic acid, iron, vitamin $A$, vitamin $\mathrm{B} 12$, or vitamin $\mathrm{C}$ while more than $50 \%$ use vitamin E. Less than $10 \%$ of the entire sample of adults with Down syndrome

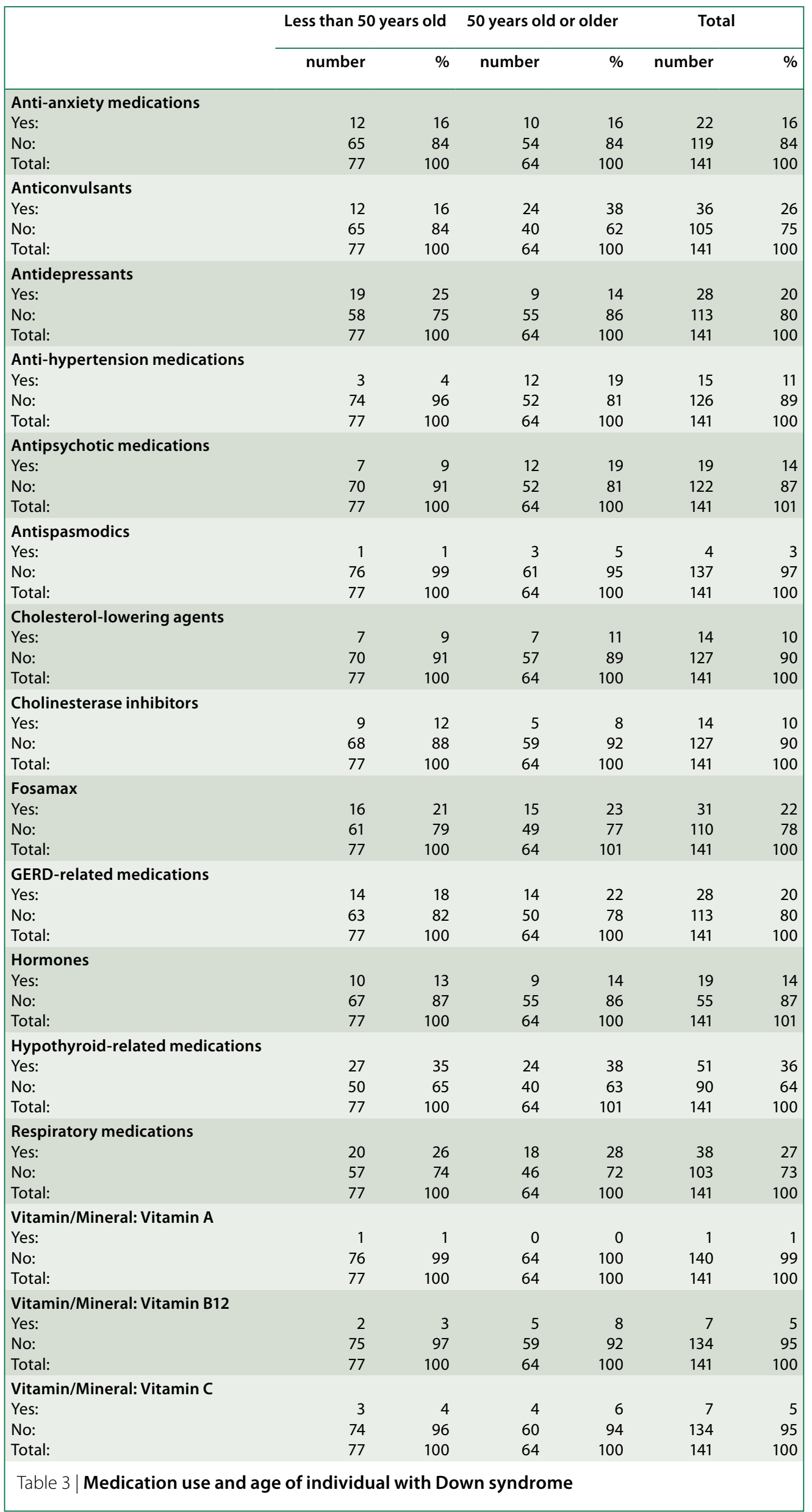




\begin{tabular}{|c|c|c|c|c|c|c|}
\hline & \multicolumn{2}{|c|}{ Less than 50 years old } & \multicolumn{2}{|c|}{50 years old or older } & \multicolumn{2}{|c|}{ Total } \\
\hline & number & $\%$ & number & $\%$ & number & $\%$ \\
\hline \multicolumn{7}{|l|}{ Vitamin/Mineral: Vitamin E } \\
\hline Yes: & 39 & 51 & 35 & 55 & 74 & 53 \\
\hline No: & 38 & 49 & 29 & 45 & 67 & 48 \\
\hline Total: & 77 & 100 & 64 & 100 & 141 & 101 \\
\hline \multicolumn{7}{|l|}{ Vitamin/Mineral: Calcium } \\
\hline Yes: & 23 & 30 & 19 & 30 & 42 & 30 \\
\hline No: & 54 & 70 & 45 & 70 & 99 & 70 \\
\hline Total: & 77 & 100 & 64 & 100 & 141 & 100 \\
\hline \multicolumn{7}{|l|}{ Vitamin/Mineral: Folic Acid } \\
\hline Yes: & 1 & 1 & 3 & 5 & 4 & 3 \\
\hline No: & 76 & 99 & 61 & 95 & 137 & 97 \\
\hline Total: & 77 & 100 & 64 & 100 & 141 & 100 \\
\hline \multicolumn{7}{|l|}{ Vitamin/Mineral: Iron } \\
\hline Yes: & 1 & 1 & 0 & 0 & 1 & 1 \\
\hline No: & 76 & 99 & 64 & 100 & 140 & 99 \\
\hline Total: & 77 & 100 & 64 & 100 & 141 & 100 \\
\hline \multicolumn{7}{|c|}{ Vitamin/Mineral: Multivitamin } \\
\hline Yes: & 23 & 30 & 24 & 38 & 47 & 33 \\
\hline No: & 54 & 70 & 40 & 63 & 94 & 67 \\
\hline Total: & 77 & 100 & 64 & 101 & 141 & 100 \\
\hline \multicolumn{7}{|l|}{ Vitamin/Mineral: None } \\
\hline Yes: & 24 & 31 & 18 & 28 & 42 & 30 \\
\hline No: & 53 & 69 & 46 & 72 & 99 & 70 \\
\hline Total: & 77 & 100 & 64 & 100 & 141 & 100 \\
\hline \multicolumn{6}{|l|}{ Vitamin/Mineral: Other } & 1 \\
\hline Yes: & 77 & 100 & 63 & 98 & 140 & 99 \\
\hline $\begin{array}{l}\text { No: } \\
\text { Total: }\end{array}$ & 77 & 100 & 64 & 100 & 141 & 100 \\
\hline \multicolumn{7}{|l|}{ Other Medication(s) } \\
\hline Yes: & 26 & 34 & 30 & 47 & 56 & 40 \\
\hline No: & 51 & 66 & 34 & 53 & 85 & 60 \\
\hline Total: & 77 & 100 & 64 & 100 & 141 & 100 \\
\hline
\end{tabular}

uses cholinesterase inhibitors. Comparable percentages of younger and older adults with Down syndrome use Fosamax (21\% vs. $23 \%$ ) and medications for gastroesophageal reflux disease (18\% vs. $22 \%$ ), hypothyroidism ( $35 \%$ vs. $38 \%$ ) and respiratory conditions ( $26 \%$ vs. $28 \%$ ).

Extremely low percentages of younger and older adults with Down syndrome use antispasmodic medications ( $1 \%$ vs. $5 \%)$. Considerably low percentages of younger adults with Down syndrome use anti-hypertension medications (4\%) as compared to older adults (19\%). Likewise, lower percentages of younger adults than older adults with Down syndrome use anticonvulsants $(16 \%$ vs. $38 \%$ ) and antipsychotic medications (9\% vs. $19 \%$ ); however, greater percentages of younger adults with Down syndrome use antidepressants as compared to older adults with Down syndrome (25\% vs. $14 \%$ ). Greater percentages of older adults with Down syndrome use multivitamins as compared to younger adults with Down syndrome (38\% vs. $30 \%$ ). Finally, other medications such as pain relievers, prophylactic antibiotics, and topical ointments are used more often by older adults with Down syndrome as compared to younger adults with Down syndrome (47\% vs. $34 \%$ ).

\section{Discussion}

Overall, our study involved 141 adults with Down syndrome. Our sample of adults with Down syndrome was approximately $60 \%$ male and $40 \%$ female. Slightly more than $75 \%$ of our sample experienced memory loss (as reported by family members or caregivers) and/or were diagnosed with dementia. This is not surprising, given that one of the primary reasons for the referral of adults with Down syndrome to the physician clinics at this metropolitan hospital setting occurs as a result of signs and symptoms of dementia in the adult patient. Nonetheless, adults with Down syndrome who are 50 years old or older constitute a greater percentage (81\%) of those with dementia as compared to younger adults $(71 \%)$. These findings are consistent with research demonstrating that memory loss and the likelihood of dementia increase with age for adults with Down syndrome.

Our study demonstrates that slightly more than $20 \%$ of adults with Down syndrome experience seizures, with greater percentages of older adults with Down syndrome diagnosed with seizure disorder (34\%) as compared to the younger group (10\%). Although the occurrence of seizures in adults with Down syndrome has been recognised for years, our data analysis suggests that (new onset) seizures may accompany the aging process.

While cardiac conditions such as murmurs (14\%) and ASVD (18\%) occur in this sample of adults with Down syndrome, extremely low percentages of hypertension (3\%) and hypercholesterolaemia (9\%) are found. In fact, coronary artery disease does not appear to occur in this sample of adults with Down syndrome. Further, due to the fact that very few adults with Down syndrome in this sample were diagnosed with diabetes mellitus, we do not have a category for the presence of diabetes mellitus. However, given the implications that diabetes mellitus has for the development of coronary artery disease, we are interested in future research that examines the individual and combined effects of diabetes mellitus, HTN, and hypercholesterolaemia on the development of coronary artery disease in adults with Down syndrome.

Relatively large percentages of older adults with 
Down syndrome (34\%) experience skin conditions such as fungal infections, psoriasis, and skin rash as compared to younger adults with Down syndrome (20\%), leading us to question whether the presence of such skin conditions is related to environment, the onset of memory loss and dementia, and poor self-care that may occur over time. Additionally, urinary incontinence is present in nearly $18 \%$ of this sample and may be related to the high percentage of skin conditions in this sample of adults with Down syndrome.

Hypothyroidism is present in over $40 \%$ of this sample of adults with Down syndrome, a finding that is consistent with prior research demonstrating the commonality with which this medical condition occurs in adults with Down syndrome. Osteoporosis occurs in about $24 \%$ of the overall sample of adults with Down syndrome. Interestingly enough, however, osteoporosis occurs more frequently in younger adults with Down syndrome (26\%) as compared to the older group (22\%). Other medical conditions that commonly occur in this sample of adults with Down syndrome include gastroesophageal reflux disease (14\%), pulmonary conditions (18\%), and obstructive sleep apnoea (19\%). Geriatricians, geriatric nurses, and other members of the health care team should be aware of the occurrence of medical conditions such as these, so as to focus assessment and treatment options as well as improve the overall quality of life for adults with Down syndrome.

Our review of medication use in this sample of adults with Down syndrome confirms the presence of thyroid supplementation, anti-anxiety and antidepressant medications, and anticonvulsants as common and appropriate. Multivitamins are used at least a third of the time, and Vitamin $\mathrm{E}$ is used by over $50 \%$ of adults in this sample. We are encouraged by the use of calcium with Vitamin D $(30 \%)$ and Fosamax (22\%) to help in maintaining bone health, thereby decreasing the risk of osteoporosis in this vulnerable group of adults.

A relationship exists between medical conditions and medications occurring in this sample of adults with Down syndrome. Overall, the majority of medications are used to treat commonly occurring medical conditions, with the exception of memory loss and dementia. Relatively little use of cholinesterase inhibitors (10\%) occurs in this sample of adults with Down syndrome; only $12 \%$ of younger adults with Down syndrome and $8 \%$ of older adults with Down syndrome use cholinesterase inhibitors. We believe that this can be explained by the fact that the use of cholinesterase inhibitors to slow the progression of memory loss in adults with Down syndrome has yet to be examined and under- stood more fully.

Further research is necessary in order to assess the natural history of commonly occurring medical conditions such as those included in our study. The fact that certain medical conditions such as cancer and hypertension appear to occur infrequently in adults with Down syndrome warrants further study; this is particularly the case when comparing samples such as these to the general population. This sort of study may include comparisons of men and women with regard to incidence and prevalence of medical conditions. Barriers to health care services and appropriate diagnosing and treatment options for adults with Down syndrome should be examined. Finally, systems of care that allow for screening and prevention of commonly occurring medical conditions such as these must be developed and implemented.

\section{Limitations}

Our study is primarily limited methodologically with regard to the cross-sectional nature of our research design and our sampling strategy. A longitudinal design that would permit the researchers to follow participants over time is ideal, particularly when considering research that applies to the aging process. The onset of certain, commonly occurring medical conditions (e.g., memory loss and dementia, hypothyroidism, seizure disorder) could be pinpointed, thereby facilitating a more thorough understanding of appropriate intervention, treatment, and cure. Further, our study is not powered for inferential statistical tests that have the potential to demonstrate significance.

In our sample of adults with Down syndrome, the point at which an adult in the " $<50$ years old" group develops dementia, hypothyroidism, or seizure disorder, for instance, is no clearer than for adults in the " $50+$ " group. We have no data pertaining to family history of hypercholesterolaemia, even though the presence of this condition may be familial to a certain extent. Likewise, we are limited in our sampling design in that we are dependent upon a convenience sample of adults with Down syndrome. This creates difficulty with representation and the comparison of our sample to the larger population of adults with Down syndrome. As a result, the ability to generalise the findings of our study is present but hindered. Overall, we believe that a study such as this that identifies commonly occurring medical conditions and medications that characterise adults with Down syndrome is a beginning step to future research that more definitively addresses the aging process for adults with Down syndrome. 
1. McCallion P, McCarron M. Ageing and intellectual disabilities: A review of recent literature. Current Opinion in Psychiatry. 2004;17:349-352.

2. Rainville CL, Sadeghi-Nejad A. Occurrence of hypothyroidism in hypothyroid children with Down syndrome. Pediatric Research. 1999;45:96A.

3. LeFaivre JF, Cohen SR, Burnstein FD, Simms C, Scott PH, Montgomery GL, Graham L, Kattos AV. Down syndrome: Identification and surgical management of obstructive sleep apnea. Plastic and Reconstructive Surgery. 1997;59:1133-1136.

4. Carmeli E, Merrick J, Kessel S, Masharawi Y, Carmeli V. Elderly persons with intellectual disability: A study of clinical characteristics, functional status, and sensory capacity. Scientific World Journal. 2003;3:298-307.

5. Head E, Lott IT. Down syndrome and beta-amyloid deposition. Current Opinion in Neurology. 2004;17:95-100.

6. Holland AJ, Hon J, Huppert FA, Stevens F, Watson P. Population-based study of the prevalence and presentation of dementia in adults with Down syndrome. British Journal of Psychiatry. 1998;172:493-498.

7. Prasher VP. Age-specific prevalence, thyroid dysfunction and depressive symptomatology in adults with Down syndrome and dementia. International Journal of Geriatric Psychiatry. 1995; 10:25-31.

8. Visser FE, Aldenkamp AP, van Huffelen AC, Kuilman M, Overweg J, van Wijk J. Prospective study of the prevalence of Alzheimer-type dementia in institutionalized individuals with Down syn- drome. American Journal on Mental Retardation 1997;101:400-412.

9. Ellis FJ. Management of pediatric cataract and lens opacities. Current Opinion in Pediatrics. 2002;13:33-37.

10. Cengiz M, Seven M, Suyugul N. Antioxidant system in Down syndrome: A possible role in cataractogenesis. Genetic Counseling. 2003;13:339-342.

11. Corsi MM, Malavazos AE, Passoni D, Licastro F. LDL receptor expression on T-lymphocytes in old patients with Down syndrome. Immunity and Ageing. 2005;2(1):3.

12. Bocconi L, Nava, S, Fogliani R, Nicolini U. Trisomy 21 is associated with hypercholesterolemia during intrauterine life. American Journal of Obstetrics and Gynecology. 1997;176:540-543.

13. Shireman RB, Muth J, Toth JP. (1988).[14C]acetate incorporation by cultured normal, familial hypercholesterolemia and Down's syndrome fibroblasts. Biochimica et Biophysica Acta: Lipids and Lipid Metabolism. 1988;958:352-360.

14. Fernhall B, Pitetti KH, Rimmer JH, McCubbin JA, Rintala P, Millar AL, Kittredge J, Burkett LN. Cardiorespiratory capacity of individuals with mental retardation including Down syndrome. Medicine and Science in Sports and Exercise. 1996;28:366-371.

15. Prasher V, Cunningham C. Down syndrome. Current Opinion in Psychiatry. 2001;14:431-436.

16. Tsiouris JA, Patti PJ, Tipu O, Raguthu, S. Adverse effects of phenytoin given for late-onset seizures in adults with Down syndrome. Neurology. 2002;59:779-780.
17. Deutsch SI, Rosse RB, Mastropaolo J, Chilton, M. Progressive worsening of adaptive functions in Down syndrome may be mediated by the complexing of soluble $A[$ beta] peptides with the[alpha]7 nicotine acetylcholine receptor: Therapeutic implications. Clinical Neuropharmacology. 2003;26:277-283.

18. Walsh PN. Ageing and mental retardation. Current Opinion in Psychiatry. 2002;15:509-514.

19. Harman D. Alzheimer's disease: Role of aging in pathogenesis. Annals of the New York Academy of Sciences. 2002;959:384-395.

20. Devenny DA, Wegiel J, Schupf N, Jenkins $E_{\text {, }}$ Zigman W, Krinsky-McHale SJ, Silverman WP. Dementia of the Alzheimer's type and accelerated aging in Down syndrome. Science of Aging Environment. 2005;14: dn1.

21. McCarron M, Gill M, McCallion P, Begley C. Health comorbidities in ageing persons with Down syndrome and Alzheimer's disease. Journal of Intellectual Disabilities Research. 2005;49:560-566.

22. Mosby's 2006 Drug Consult for Nurses (p. 1177). St. Louis, MO: Elsevier Mosby.

Received: 1 November 2006; Accepted 14 February 2007; Published online: 21 August 2007. 